relative motion is also inertial and the transformation between them is then a Newtonian approximation to a Lorentz transformation. But there is no preferred set of frames in the absolute sense of special relativity to start with, because, once gravitational fields are allowed, there is no precise set of forces and any frame of reference can be considered inertial so long as we are prepared to introduce sufficiently strange gravitational fields. The only sense in which the six-fold infinity of frames can be said to be preferred is that of convenience.

The basis of Dingle's long-standing arguments with the relativists seems to be his insistence on the arbitrary nature of inertial frames. Neither his antagonists nor he himself have noticed that this arbitrariness is explicitly denied. The current discussion makes all this a little clearer.

C. W. Kilmister

\section{Element synthesis and isotope anomalies in the early Solar System}

from Grenville Turner

THE chemical elements which make up the Solar System were synthesised by various nuclear processes which occur at different stages in the life cycle of stars. Much of the experimental evidence which verifies in detail the operation of these processes is to be found in elemental and isotopic abundances within the Earth, meteorites and recently, the Moon. Many types of process are known to occur and there are different stellar environments appropriate to each; yet significantly, isotopic abundances are, with very few exceptions, within error uniform throughout those parts of the Solar System so far sampled. This observation implies that the material which came together to form the Solar System was at a very early stage homogenised to a very high degree.

Most of the exceptions to this rule (referred to as isotope anomalies) can be understood in terms of the radioactive decay of some long lived precursor isotope and provide the means of determining such things as the age of the Solar System and the duration of some of the element-building processes. Other exceptions in meteorites and lunar samples can be understood in terms of currently operating nuclear processes induced by cosmic rays. These processes are well understood.

Recently two isotope anomalies have been discovered in the very primitive Allende meteorite. Neither anomaly is currently understood but they may result from nuclear processes operating immediately prior to or during Solar System formation. The first of these anomalies to be found (Clayton et al., Science, 182, 485; 1973) occurs in oxygen in A1-rich inclusions present in Allende. Correlated variations in $\left({ }^{: 8} \mathrm{O} /{ }^{16} \mathrm{O}\right)$ and $\left({ }^{17} \mathrm{O} /{ }^{16} \mathrm{O}\right)$ ratios can be most easily understood in terms of the presence of variable amounts of the single isotope ${ }^{16} \mathrm{O}$. Whether this is the correct interpretation or not it seems difficult to escape the conclusion that one is seeing in Allende the results of the incomplete mixing of the products of nuclear synthesis.

The second recent observation concerns the isotope ${ }^{26} \mathrm{Mg}$. ${ }^{26} \mathrm{Mg}$ is the daughter product of ${ }^{26} \mathrm{Al}$, an isotope which has aroused much speculation as a possible heat source during the early history of the Solar System. The need for a heat source capable of heating planetary objects on a rapid time scale arises from a number of arguments. Chemical arguments based on the presence in meteorites and the Earth of volatile elements suggest that solid material originally condensed at relatively low temperatures to form the Solar System. In contrast there is much evidence that a number of processes requiring high temperatures occurred within the first hundred million years of Solar System history; for example, the melting of iron to form iron meteorites, and magmatic differentiation of rocks on the Moon and in certain classes of stone meteorites. The heat source responsible is currently a matter for speculation, ${ }^{26} \mathrm{Al}$ being only one of a number of possibilities.

Stimulated partly by the search for a heat source several attempts have been made to search for the ${ }^{26} \mathrm{Mg}$ anomalies which would result from in situ decay of ${ }^{26} \mathrm{Al}$. Two factors make the search a difficult one. First of all ${ }^{26} \mathrm{Al}$ has a geologically short half life. $7.4 \times 10^{5} \mathrm{yr}$, and so the search must be confined to the most primitive of meteorites to have any chance of succeeding. Second, $\mathrm{Mg}$ is itself an abundant element and the anticipated anomalies would be undetectable in all minerals but those most poor in $\mathrm{Mg}$ and rich in $\mathrm{Al}$. The search for ${ }^{26} \mathrm{Mg}$ anomalies has had a chequered career and ar early claim to have detected them (Clark et al., J. geophys. Res., 75, 448; 1970) was later shown by an exhaustive series of measurements to have been incorrect (Schramm et al., Earth. planet. Sci. Lett., 10, 44; 1970).

A recent issue of Nature contains one of the latest additions to the saga. Gray and Compston (Nature, 251, 495; 1974) have continued the search in the Al-rich inclusions of Allende, following the observation of the ${ }^{16} \mathrm{O}$ anomalies and encouraged by $\mathrm{Sr}$ isotope data which indicate that these inclusions are the most primitive material known to man (Gray et al., Icarus, 20, 213; 1973). After a series of measurements, on the meteorite and three inclusions, they are able to report a small but significant anomaly for one of the inclusions which would correspond to an $0.4 \%$ excess of ${ }^{26} \mathrm{Mg}$. The $(\mathrm{Al} / \mathrm{Mg}$ ) ratios of the other two inclusions are less favourable and an anomaly, if present at an equivalent level, would be barely detectable. Gray and Compston conclude that if the anomaly is the result of in situ ${ }^{26} \mathrm{Al}$ decay then the implied initial concentration of ${ }^{26} \mathrm{Al}$ would represent a significant early Solar System heat source.

A second group of research workers have been searching for $\mathrm{Mg}$ anomalies in Allende and their results have also been published recently (Lee and Papanastassiou, Geophys. Res. Lett., 1, 227 ; 1974). This work, coming from the laboratory which painstakingly laid to rest the early claims of ${ }^{26} \mathrm{Mg}$ anomalies, indicates that, in Allende at least, anomalies are present. A most significant finding is however that in one of the samples the anomaly is negativewhich appears to rule out in situ decay of ${ }^{26} \mathrm{Al}$ as the cause of the anomalies.

Lee and Papanastassiou have performed 39 analyses on 21 distinct samples from Allende and found anomalies in nine of the samples. The anomalies are found in the Al-rich inclusions and expressed in terms of the $\left({ }^{26} \mathrm{Mg} /\right.$ ${ }^{24} \mathrm{Mg}$ ) ratio range from $-0.17 \%$ to $+0.30 \%$ with typical uncertainties of around $0.02 \%$. The shifts are much smaller than those found in oxygen, which range up to $5 \%$. Moreover there seems to be no direct correlation with the oxygen anomalies in that the samples with large oxygen anomalies show no magnesium anomaly. There is also no correlation with $(\mathrm{Al} / \mathrm{Mg})$ ratio and this in addition to the negative ${ }^{26} \mathrm{Mg}$ anomaly referred to above argues against in situ decay of ${ }^{26} \mathrm{Al}$. The authors suggest a number of possible nucleosynthetic processes which could lead to enrichment or depletion of various $\mathrm{Mg}$ isotopes but argue that the absence of a correlation with the oxygen anomalies hinders the identification of a single process as the cause.

In summary there is now a growing body of evidence for the presence in meteorites of the products of nucleosynthesis which have not been thoroughly mixed. To the $\mathrm{O}$ and $\mathrm{Mg}$ anomalies should be added two as yet unexplained anomalies in the inert gases $\mathrm{Xe}$ and $\mathrm{Ne}$ (Black. Geochim. cosmochim. Acta., 36, 377; 1972). The explanation of these effects is a challenge which, if met, will add greatly to our understanding both of the processes of nucleosynthesis and of the early evolution of the Solar System. 